\title{
Marketing y publicidad en el aula de ELE. Una propuesta didáctica
}

\author{
CRISTINA ViLlalba IBÁÑEZ \\ Universtitat Jaume I / Universitat de València \\ cristina.villalba@uv.es
}

\begin{abstract}
Resumen: El objetivo de este trabajo es presentar una propuesta didáctica que se enmarca dentro de la enseñanza del español para fines específicos (EFE) y, más concretamente, del español para los negocios. A partir de un enfoque comunicativo por tareas, se propone que el alumnado diseñe una campaña de publicidad de un producto. Para lograr este objetivo, los estudiantes se organizan en grupos de trabajo de entre $4 \mathrm{y}$ 6 personas y deben realizar, en las distintas sesiones de trabajo, las siguientes actividades: elegir el producto que van a promocionar, identificar y hacer un perfil del público objetivo y el buyer persona de dicho producto, reflexionar sobre la mejor ubicación, elaborar un anuncio gráfico y, finalmente, presentar el producto ante la clase. Esta propuesta ha sido implementada en un curso de la asignatura de Español para los Negocios con gran aceptación por parte del alumnado.
\end{abstract}

Palabras clave: español para los negocios, español para fines específicos, EFE, marketing, publicidad, ELE

\section{Marketing and publicity in the Spanish as Foreign Language classroom. A didactic proposal.}

Abstract: The aim of this work is to present a didactic proposal within the frame of the teaching of Spanish for Specific Purposes and, more specifically, Spanish for Business. From a communicative approach through the task-based methodology, we propose students to design an advertising campaign for a product. To achieve this goal, the students are organized into work groups of between 4 and 6 people. They must carry out, in the different work sessions, the following activities: to select a product for their campaign, to identify and make a profile of the target audience and the buyer persona of the product, to reflect on the best localization, to elaborate a graphic advertisement and, finally, to present the product to the class. This proposal has been implemented in a course of the subject of Spanish for Business with great acceptance among the students

Keywords: Spanish for Business, Spanish for Specific Purposes, Marketing, Publicity, SFL

\section{Introducción}

En un mercado cada vez más global donde las economías hispanoamericanas juegan un papel importante, la necesidad de aprender español para poder desenvolverse en entornos de negocios es una realidad. Por ello, cada vez es más frecuente encontrar en las librerías, en los encuentros de profesores o en la red, recursos didácticos para trabajar en el aula de español para los negocios. En este sentido, este artículo pretende ser una contribución más orientada a trabajar aspectos relacionados con el marketing y la publicidad partiendo del enfoque comunicativo mediante tareas. 


\title{
2. Marco teórico
}

\subsection{Español para fines específicos y español para los negocios}

A partir de la segunda mitad del siglo XX, el contexto en el que se produce la comunicación suscitó el interés de los lingüistas, hecho que dio lugar a la creación de nuevas disciplinas, como por ejemplo, la pragmática. En lo que respecta a la enseñanza y el aprendizaje de lenguas, se originó una profunda reflexión sobre qué tipo de lengua enseñar y cómo hacerlo, pues la lengua como instrumento de comunicación debía concebirse dentro de un marco contextual. Es en este momento cuando la enseñanza de lenguas para fines específicos comienza a desarrollarse.

De acuerdo con Widdowson (1983: 5-7), la lengua general busca adquirir un grado de competencia genérico, de manera que los usuarios sean capaces de desarrollarse en varios contextos. Por el contrario, la lengua asociada a un fin específico está acotada a contextos concretos y a tareas claramente definidas. En relación con esto, el diseño de toda propuesta didáctica para fines específicos:

\begin{abstract}
debe centrarse en el establecimiento de las condiciones adecuadas para que los estudiantes adquieran y desarrollen la competencia comunicativa (gramatical, discursiva, sociolingüística, estratégica y sociocultural) que requiere su actividad profesional, esto es, las capacidades, habilidades y técnicas de comunicación que hay que aplicar en un contexto determinado. (Beltrán, Lobato, y Gargallo, 2012: 277-278)
\end{abstract}

En el caso del mundo de los negocios, buena parte de las interacciones se desarrollan en entornos formales. Se espera que una persona comunicativamente competente sea capaz de llevar a cabo una entrevista, elaborar un informe, participar en una reunión de trabajo, abrir un negocio o vender un producto, por citar algunos ejemplos. Así, la capacidad de satisfacer estas expectativas necesariamente implica abordar una serie de contenidos y destrezas relacionadas con la competencia comunicativa bajo el prisma del mundo empresarial y el comercio.

\subsection{El enfoque por tareas: una metodología próxima y motivadora}

$\mathrm{Si}$, por lo general, el proceso de adquisición de segundas lenguas tiene que ser activo y práctico para que el aprendizaje sea significativo, en el caso de español para los negocios esto fundamental, puesto que se trata de un lenguaje aplicado a contextos concretos.

En esta línea, el enfoque por tareas se revela como una metodología que se adapta a las características de la clase de español para los negocios, pues subraya la necesidad de generar situaciones de comunicación que favorezcan el uso real de la lengua en el aula (Juan Lázaro, 2003). Una de las claves en este enfoque es que el diseño de las unidades didácticas debe comenzarse a partir de un análisis de los intereses y necesidades del alumnado. En el caso de la enseñanza de lenguas para fines específicos, hay que pensar en qué contextos y con qué finalidad van a comunicarse los aprendices para establecer, primeramente, la tarea que deben desarrollar en la unidad. Una vez que ya se ha concretado la tarea final y los objetivos que debe alcanzar el alumnado, es el momento de abordar los contenidos concretos que se van a trabajar en la unidad y diseñar las actividades (Estaire, 2011).

La tarea que deben desarrollar los estudiantes, de acuerdo con Estaire y Zanón (1990:63), debe cumplir cinco características: 
1. Representativa de procesos de comunicación reales.

2. Identificable como unidad de actividad en el aula.

3. Dirigida intencionalmente al aprendizaje del lenguaje.

4. Diseñada con un objetivo, estructura y unidad de trabajo.

5. Orientada a la consecución de un objetivo de manipulación de información/significados.

En el caso de nuestra propuesta, la tarea final que debe desarrollar el alumnado consiste en presentar una campaña de publicidad sobre un producto que hayan escogido promocionar. Esta actividad es una parte fundamental del comercio y de nuestra vida diaria, pues si bien es cierto que no toda la población elabora campañas de publicidad, sí que es su destinataria en algún momento. De este modo, la tarea final conecta directamente con el entorno próximo de los estudiantes.

\section{Propuesta didáctica: ;Me lo quitan de las manos!}

La propuesta didáctica que se presenta a continuación ha sido diseñada para la asignatura Español para los Negocios, que se imparte en la Universitat de València. Se trata de una asignatura semestral, con dos sesiones de una hora y cuarenta y cinco minutos a la semana. Esta asignatura está orientada a estudiantes internacionales que cursan al menos un semestre en España como parte de su formación.

\subsection{Caracterización del alumnado y agrupaciones}

Pese a que se ha señalado que una de las ventajas de la enseñanza de español de los negocios es que cuenta con un alumnado con conocimientos sobre el mundo empresarial que puede facilitar la adquisición del léxico (Villarrubia Zúñiga, 2007), lo cierto es que la interdisciplinariedad (cada vez más presente en los planes de estudio) hace que aprendices con distintos perfiles se interesen por estos cursos de español. Así, todos nuestros estudiantes participan en un programa de estudios internacional y sus edades suelen estar comprendidas entre los 19 y los 23 años, pero existen dos factores que los diferencian: el nivel de español y el grado del que provienen.

En cuanto al nivel de lengua, se espera que los participantes ingresen en el curso con un nivel B2 de acuerdo con el MCER. No obstante, no todos los estudiantes se adscriben a ese nivel o alcanzan ese nivel en todas las destrezas. En otros casos, el alumnado puede superar el nivel exigido pero carecer de los conocimientos y las destrezas particulares del español de los negocios.

Por lo que respecta a los estudios, generalmente provienen de grados afines al mundo de los negocios, como pueden ser Economía, Finanzas o Administración y Dirección de Empresas. Con todo, no es extraño encontrar estudiantes de Ingeniería, Traducción o Filología, que necesitan inscribirse en esta asignatura porque así se lo exigen en sus planes de estudio.

En definitiva, puede concluirse que el alumnado está interesado en aprender español, pero no todos tienen igual de desarrollada la competencia comunicativa en esta lengua ni tienen el mismo interés por aprender una lengua de especialidad. Por ello, se hace imprescindible trabajar a partir de agrupaciones donde esa heterogeneidad se convierta en una ventaja para los aprendientes. 
Para nuestra propuesta didáctica, los alumnos deben organizarse en grupos de entre 4 y 6 personas. Esta agrupación puede ser aleatoria o puede estar organizada por el docente en base a las motivaciones y el grado de competencia del alumnado. En ambos casos se trata de unir a personas con distintos niveles, intereses o conocimientos sobre el mundo de los negocios para favorecer la cooperación.

\subsection{Objetivos y contenidos}

Ya se ha explicado que el alumnado debe elaborar una campaña publicitaria para un producto como tarea final. Los objetivos derivados de esa tarea pueden resumirse en los siguientes puntos:

1. Elaborar un DAFO.

2. Ofrecer y negociar su punto de vista para elegir el producto de la campaña.

3. Hacer hipótesis relacionadas con las posibilidades comerciales de un producto.

4. Diseñar un anuncio gráfico.

5. Presentar oralmente una campaña de publicidad ante la clase.

6. Valorar las campañas publicitarias de los compañeros.

En relación con estos objetivos, los contenidos que se van a trabajar en esta propuesta didáctica se resumen en la siguiente tabla:

\begin{tabular}{|c|c|c|c|}
\hline $\begin{array}{l}\text { Contenidos } \\
\text { funcionales }\end{array}$ & $\begin{array}{l}\text { Contenidos } \\
\text { gramaticales }\end{array}$ & Contenidos léxicos & $\begin{array}{l}\text { Contenidos } \\
\text { socioculturales }\end{array}$ \\
\hline $\begin{array}{l}\text { Dar la opinión (desde } \\
\text { mi punto de vista, a } \\
\text { mi parecer...) } \\
\text { Hacer hipótesis (tal } \\
\text { vez, probablemente, a } \\
\text { lo mejor...) }\end{array}$ & $\begin{array}{l}\text { Ser y estar + adjetivo } \\
\\
\text { Uso de indicativo o } \\
\text { subjuntivo para } \\
\text { indicar probabilidad. } \\
\text { Imperfecto } \\
\text { subjuntivo de } \\
\text { condicional } \\
\text { abriéramos } \\
\text { negocio, } \\
\text { tendriamos...) }\end{array}$ & 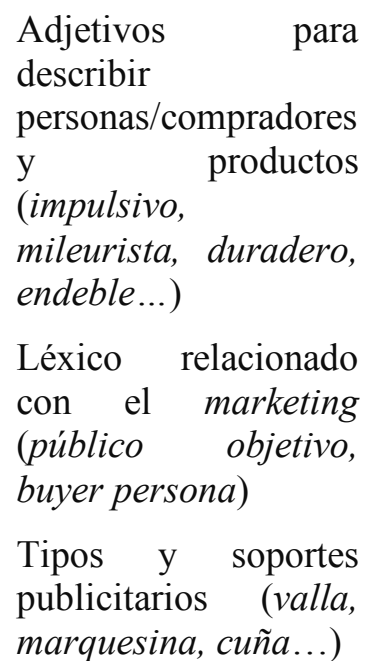 & $\begin{array}{l}\text { Zonas comerciales en } \\
\text { Valencia. } \\
\text { Hábitos de consumo. }\end{array}$ \\
\hline
\end{tabular}

Tabla 1. Contenidos de la propuesta didáctica.

Estos contenidos se abordan a lo largo de las sesiones de trabajo que comprende nuestra unidad didáctica.

\subsection{Secuenciación}

Las actividades que conforman esta propuesta se distribuyen en cuatro sesiones de trabajo en el aula. Durante la primera sesión, se forman los grupos de trabajo de manera 
aleatoria, se presenta la dinámica de trabajo y el calendario (imagen 1) y se desarrollan los dos primeros puntos de la campaña publicitaria: la elección del producto y la elaboración de un DAFO.

Para la sesión dos, el grupo tiene que elaborar un breve informe sobre cuál es su público objetivo y escribir el perfil de su buyer persona. En esta sesión también se introduce una primera reflexión sobre la localización.

En la sesión tres, el grupo debe decidir cuál es la mejor ubicación para vender su producto y diseñar un anuncio publicitario gráfico. También se trabajan los distintos tipos y soportes publicitarios.

Por último, en la sesión final deben presentar el trabajo realizado en las sesiones anteriores ante sus compañeros para promocionar su producto. A su vez, el resto de estudiantes deben valorar las presentaciones de sus compañeros.

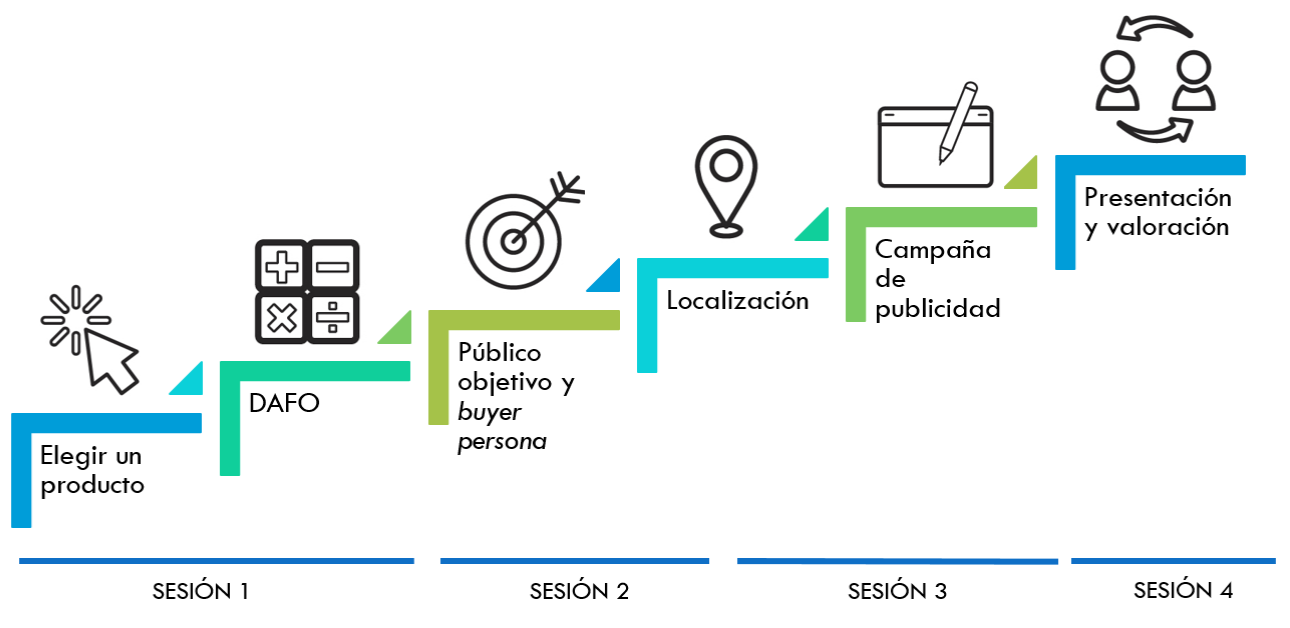

Imagen 2. Dinámica de trabajo y calendario.

\subsection{Actividades}

\subsubsection{Sesión 1}

Una vez que los grupos se han formado aleatoriamente o el docente ha ordenado los grupos de manera heterogénea, podemos comenzar con una actividad de calentamiento destinada a generar curiosidad y motivación por la tarea. Esta actividad también ayuda a que los miembros del grupo se conozcan. Para ello, se propone que respondan a unas preguntas por parejas y luego las pongan en común con el resto de miembros de su grupo, como se ilustra en la Imagen 2. 


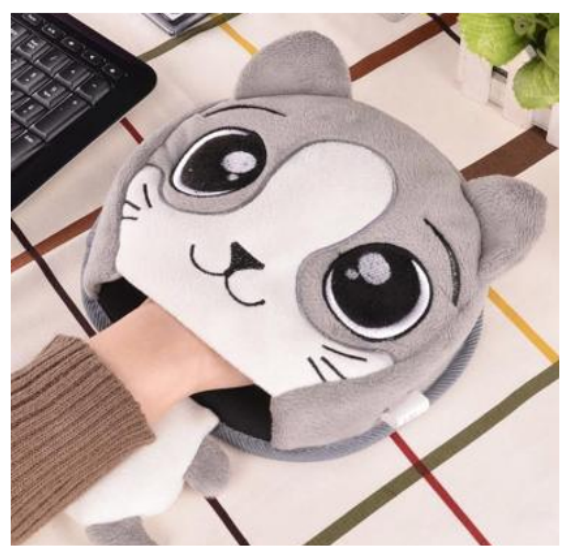

\section{Por parejas, responded a las siguientes preguntas:}

a. ¿Conoces la expresión me lo quitan de las manos? ¿Qué crees que significa?

b. ¿Cuál es el objeto más extraño que has comprado? ¿Por qué lo compraste? ¿Sigues contento/a con esa compra?

c. ¿̇Para qué creéis que puede servir este objeto? ¿̇Lo compraríais? ¿̇Por qué?

Imagen 2. Actividad de calentamiento.

Seguidamente, se pide que, de manera individual, cada miembro del grupo proponga un objeto para el proyecto que desarrollaremos en las siguientes sesiones. Dependiendo de la creatividad del grupo, pueden diseñar ellos mismos aquello que desean promocionar o buscar en la red algún producto que pueda resultar curioso o interesante ${ }^{1}$.

Cuando ya han hecho la selección del producto, se les pide que elaboren individualmente un DAFO. El DAFO es una herramienta de análisis para identificar las Debilidades, Amenazas, Fortalezas y Oportunidades de una empresa, un producto o un candidato, por poner algunos ejemplos. Puesto que no todos los estudiantes están familiarizados con esta herramienta, primeramente se hace un DAFO grupal del producto que han visto en la actividad de calentamiento y luego se les invita a que lo hagan del producto que han escogido. Para finalizar la sesión, tienen que presentar a sus compañeros el DAFO y el producto seleccionado para votar cuál se trabajará durante las sesiones posteriores.

\subsubsection{Sesión 2}

En la sesión 2 los miembros del grupo tienen que reflexionar sobre cuál es su público objetivo, es decir, el conjunto de (potenciales) consumidores a quienes debemos dirigir nuestra campaña publicitaria. El público objetivo suele comprender grandes segmentos de la población, de manera que para perfilarlo se tienen en cuenta factores demográficos como el sexo, la edad, la ubicación, la formación académica o el poder adquisitivo (Schüler 2017). Aunque el marketing tradicional partía de este concepto, la aparición de nuevas formas de marketing en el entorno digital han derivado en la elaboración de perfiles más personales e humanizados de sus potenciales clientes: los buyer persona. El buyer persona es una representación ficticia de un cliente ideal. Para poder desarrollar

\footnotetext{
${ }^{1}$ Para esta actividad en concreto, recomiendo entrar en <www.odditymall.com $>$ por la variedad y rareza de los productos que ofrecen. El único inconveniente es que la página todavía no está disponible en español y los contenidos se presentan en inglés. No obstante, esto es un aliciente para las personas cuyo nivel de español es inferior al esperado y necesitan una ayuda para entrar en la dinámica del proyecto.
} 
este perfil, se tienen en cuenta las características que determinan el público objetivo y se añaden, además, aspectos personales como motivaciones, retos, preocupaciones $\mathrm{u}$ objetivos vitales (Siqueira 2018). La finalidad última de la creación del buyer persona es asegurarse de que las herramientas de marketing (como la publicidad) llegan a los consumidores que verdaderamente pueden estar interesados en adquirir un producto.

Dentro del aula de español para los negocios, trabajar en la elaboración del público objetivo y el buyer persona nos permite desarrollar las destrezas orales, pues el alumnado debe imaginar cómo se concretan estos conceptos en relación con su producto, al tiempo que practican las destrezas escritas, pues deben completar un breve formulario y describir su buyer persona.

\section{Nuestro Público Objetivo}

\begin{tabular}{|l|l|}
\hline Género & $\begin{array}{l}\text { Ambos sexos. Este producto no es específico de } \\
\text { género pero más para las mujeres }\end{array}$ \\
\hline Rango de edad & $\begin{array}{l}25-30 \text { (mujeres) porque tendrán un poco más } \\
\text { dinero pero son todavía en la edad de utilizar los } \\
\text { redes sociales como facebook/ instagram y } \\
\text { quieren compartir sus creaciones. }\end{array}$ \\
\hline Promedio de ingresos mensuales & $\begin{array}{l}\text { El precio de este producto es de 19,99 €. Este } \\
\text { precio refleja un consumidor con altos ingresos } \\
\text { disponibles. Como este producto presenta un alto } \\
\text { grado de elasticidad de precios, los consumidores } \\
\text { que normalmente estarian interesados en } \\
\text { comprar este producto deberian tener un ingreso } \\
\text { disponible más alto que el promedio, el producto } \\
\text { no es "una compra esencial", es más un capricho. } \\
\text { (€2500 y más) }\end{array}$ \\
\hline Nivel de instrucción & $\begin{array}{l}\text { Graduados de la universidad que se ubican en } \\
\text { una situación financiera más estable }\end{array}$ \\
\hline Otros: & \multicolumn{2}{|l}{} \\
\hline
\end{tabular}

\section{Buyer Persona}

Maria es mujer, tiene 25 años, es graduada y vive en su casa en Valencia. Le gusta a pasar el tiempo en su hogar e invitar amigos para el café. Su pasión es servir sus favoritos cafés y pasteles con decoraciones y poner fotos preciosos en Instagram y otros redes sociales, por eso los cafés siempre deberían parecer perfectos. Como es la representante de sociedad modema, siempre busca por nuevas tecnologías y quiere poseer novedades del mercado. Sigue navegando por Internet y compra las cosas que "los influencers" le aconsejan en sus blogs.

Imagen 3. Muestra de alumnos con la descripción de su público objetivo y su buyer persona.

Estas actividades son también una buena oportunidad para trabajar vocabulario relacionado con los clientes y para repasar los usos de ser y estar.

Para concluir la sesión, se les pide que reflexionen sobre la importancia de la ubicación de una tienda y en qué zona de la ciudad les gustaría que se comercializara su producto. Esta reflexión sirve de preludio de lo que se va a trabajar en la sesión 3.

\subsubsection{Sesión 3}

La penúltima sesión está destinada a decidir la ubicación del negocio en la que se va a vender el producto seleccionado y a diseñar la campaña de publicidad. 
A partir del vídeo Claves para ubicar tu local ${ }^{2}$, el alumnado debe responder a varias preguntas destinadas a darle a conocer los principales factores que debe tener en cuenta para elegir la ubicación de un negocio. Seguidamente, se ofrecen tres puntos de venta con el fin de que los estudiantes, mediante el uso del condicional, hagan hipótesis sobre cuál sería el mejor local para vender su producto.

Luego, se les pide que elaboren un anuncio gráfico para promocionar su producto, teniendo en cuenta su público objetivo y su buyer persona, Para aumentar la motivación, se les recuerda que tendrán que exponer su campaña de publicidad ante sus compañeros para que estos la juzguen.

\subsubsection{Sesión 4}

La sesión final tiene como objetivo la presentación de la campaña publicitaria. Por una parte, los miembros del grupo tienen que presentar su producto de una manera atractiva, mostrar su DAFO, explicar quién es su público objetivo y su buyer persona, compartir la ubicación que han elegido para vender su producto y comentar el anuncio gráfico que han diseñado. Además, para involucrar al resto de la clase y favorecer un ambiente lúdico, se les pide que incluyan una diapositiva al final en la que aparezca una pregunta: «¿Cuánto crees que puede costar?».

Por otra parte, se pretende que los estudiantes que están entre el público adopten una escucha activa de lo que exponen sus compañeros. Para favorecerlo, se les pide que rellenen un pequeño cuestionario donde tienen que contestar si les interesa el producto y por qué y se les pide que pongan un precio al producto que han presentado. Esto permite generar un espacio lúdico, dado que compiten por ver quién se aproxima más al precio original del producto.

\subsection{Evaluación}

La metodología basada en el enfoque por tareas concibe el aprendizaje como un proceso. En este sentido, además de evaluar la tarea final, es decir, la presentación de la campaña de publicidad del producto, también se ha querido reconocer la asistencia a clase (pues es fundamental para realizar la tarea final) y el desarrollo de actividades en el aula que agrupan en un portafolio.

En nuestro caso, se ha dado más peso a la tarea final, que constituye el $50 \%$ de la nota de esta unidad didáctica. El $30 \%$ se corresponde con la entrega de un portafolio donde el profesorado puede revisar, de manera más detenida, el trabajo realizado por los grupos en clase. Finalmente, el $20 \%$ de la nota restante se destina a reconocer la asistencia y la participación en el aula.

\section{Conclusión}

El enfoque por tareas aplicado a la enseñanza del español y, más concretamente, orientada a las lenguas de especialidad permite generar situaciones comunicativas en el aula que favorecen el aprendizaje. En el caso de la propuesta que se ha presentado aquí, los estudiantes mostraron una actitud positiva y, en algunos casos, la vinculación con el proyecto sobrepasó las expectativas de la tarea. Por ejemplo, un grupo diseñó un

\footnotetext{
${ }^{2}$ Disponible en $<$ https://www.youtube.com/watch?v=r_HsQrQ1Tr0 $>$.
} 
logotipo para comercializar su producto y otro creó una cuenta en una red social para recopilar información de su público objetivo.

Por último, quizás el aspecto más interesante de este enfoque, al menos desde nuestro punto de vista, es que favorece el trabajo colaborativo, de manera que se crea una necesidad constante y real de comunicarse en español.

\section{Bibliografía}

Aguirre Beltrán, Blanca, SÁnchez Lobato, Jesús y SAntos Gargallo, Isabel (2012). «La enseñanza-aprendizaje del español para fines específicos: marco general». En S. Robles Avila y J. SÁNCHEZ LOBATO (Eds.); Teoría y práctica de la enseñanzaaprendizaje del español para fines específicos (pp. 275-300). Recuperado el 25/04/2019 de: https://dialnet.unirioja.es/servlet/articulo? codigo $=4510793$

ESTAIRE, Sheila (2011). «Principios básicos y aplicación del aprendizaje mediante tareas», MarcoELE: Revista de Didáctica Español Lengua Extranjera, (12), 5.

ESTAIRE, Sheila y ZANÓN, Javier (1990). «El diseño de unidades didácticas en L2 mediante tareas: Principios y desarrollo», Comunicación, Lenguaje y Educación, 7(8), $55-90$.

JUAN LÁZARO, Olga (2003). «El enfoque por tareas y el español de los negocios: integración de destrezas». En Actas del Segundo Congreso Internacional de Español para Fines Específicos (pp. 207-219). Recuperado el 10/04/2019 de https://cvc.cervantes.es/ensenanza/biblioteca_ele/ciefe/pdf/02/cvc_ciefe_02_0019.pdf

SIQUEIRA, André (16-10-2018). «Buyer Persona: ¿Qué es y por qué es importante para tu estrategia?». En Blog de Marketing Digital de Resultados. Recuperado el 12/12/2018 de https:/www.rdstation.com/blog/es/buyer-persona/

SCHÜLER, Lia (25-05-2017). «Público objetivo, cliente ideal y buyer persona: ¿cuáles son las diferencias?». En Blog de Marketing Digital de Resultados. Recuperado el 12/12/2018 de https://www.rdstation.com/blog/es/publico-objetivo-cliente-ideal-buyerpersona/

VILLARRUBiA ZÚÑIGA, Marisol (2007). «Español para fines específicos. El marco para la elaboración de un curso de español de los negocios». En S. M. Saz (Ed.), XLI Congreso de la Asociación Europea de Profesores de Español (AEPE) 125 del nacimiento de Picasso en Málaga (pp. 189-199). Recuperado el 10/04/2019 de https://cvc.cervantes.es/ensenanza/biblioteca_ele/aepe/congreso_41.htm

Widdowson, Henry G. (1983): Learning Purpose and Language Use. Oxford University Press. 